\title{
A huge malignant peripheral nerve sheath tumor with hepatic metastasis arising from retroperitoneal ganglioneuroma
}

\author{
Z.H. MENG ${ }^{1}$, Y.S. YANG ${ }^{1}$, K.L. $\mathrm{CHENG}^{2}$, G.Q. $\mathrm{CHEN}^{3}$, L.P. WANG ${ }^{3}$ and W. LI ${ }^{1}$ \\ Departments of ${ }^{1}$ Hepatobiliary-Pancreatic Surgery, ${ }^{2}$ Radiology and ${ }^{3}$ Pathology, \\ The Third Hospital of Jilin University (China-Japan Union Hospital), Changchun 130033, P.R. China
}

Received June 11, 2012; Accepted September 5, 2012

DOI: $10.3892 / \mathrm{ol} .2012 .961$

\begin{abstract}
Ganglioneuromas (GNs) are the rarest and most benign of the neuroblastic tumors. We experienced a case of huge retroperitoneal GN which differentiated into malignant peripheral nerve sheath tumors (MPNST) with hepatic metastasis. The tumor was located in the upper right quarter of the abdomen and pressed the right lobe of the liver, which was initially misdiagnosed as a liver carcinoma. The tumor shared blood supply with the right liver lob and had rich blood supplies from the abdominal aorta, renal artery and hepatic artery. It was also associated with skin pigment and recurrence shortly following resection. Our finding demonstrated that MPNST is a potent invasive malignant tumor and metastasis earlier with very poor prognosis.
\end{abstract}

\section{Introduction}

Ganglioneuromas (GNs) are typically rare benign tumors primarily arising from the central or peripheral autonomic nervous system, particularly the sympathetic system. The most affected anatomical sites are the posterior mediastinum, retroperitoneum, adrenal gland and soft tissue of the head and neck.

GNs rarely differentiate into malignant and metastasic disease (1) and differentiation into malignant peripheral nerve sheath tumors (MPNST) is extremely rare and scarcely reported. To date, there are less than 20 reported cases of MPNST worldwide in the English-language literature (2). MPNST is most frequently observed in adult life, and is slightly more common in males compared with females.

Correspondence to: Professor Wei Li, Department of Hepatobiliary-Pancreatic Surgery, The Third Hospital of Jilin University (China-Japan Union Hospital), 126 Xiantai Street, Changchun 130033, P.R. China

E-mail: weili888@hotmail.com

Dr Liping Wang, Department of Pathology, The Third Hospital of Jilin University (China-Japan Union Hospital), 126 Xiantai Street, Changchun 130033, P.R. China

E-mail: wangliping63@163.com

Key words: malignant peripheral nerve sheath tumor, liver metastasis
The diagnosis of MPNST is difficult. Surgical resection is the primary treatment of MPNST, which is usually followed by a poor outcome. MPNST occurs most frequently in the head and neck or the upper extremities and only $1 \%$ of cases have been identified in the retroperitoneum (3). The clinical features of MPNST, including the symptoms, diagnosis, pathway of malignancy transformation and characteristics of metastasis, are largely unknown and remain to be investigated. In the current study, we present a case of a huge MPNST with hepatic metastasis originating from retroperitoneal GN in a 43-year-old male, which was initially diagnosed as hepatocellular carcinoma due to the tumor's location and unusual blood supply. The study was approved by the Ethics Committee of China-Japan Union Hospital of Jilin University, Changchun, China. Written informed consent was obtained from the patient.

\section{Case report}

A 43-year-old man was admitted to The Third Hospital (ChinaJapan Union Hospital) of Jilin University (Changchun, China) due to complaints of upper abdominal pain. No other symptoms, including fever, jaundice and weight loss, were noted, but scattered brown pigments were observed on the right hypochondriac region of the abdomen (Fig .1). Ultrasonography identified a giant 'hepatic neoplasm' $25 \times 15 \times 15 \mathrm{~cm}$ in size that occupied the patient's right liver lobe and contained cystic and solid components. A contrast-enhanced computed tomography (CT) scan revealed a huge non-homogeneous, oblong shaped and relatively well-defined expansive mass occupying the right posterior liver lobes. It was enhanced in arterial phases and particularly enhanced in portal venous phases, but the inferior hepatic vena cava was not clearly presented (Fig. 2A). The 3D CT vessel reconstruction demonstrated that the majority of the tumor was nourished by the renal artery and another vessel branching from the abdominal aorta, while the top part of the tumor (4 $\mathrm{cm}$ in diameter) was nourished by the right hepatic artery (Fig. 2B). The serum tumor markers for $\alpha$-fetoprotein (AFP), carcinoembryonic antigen (CEA) and carbohydrate antigen 19-9 (CA19-9) were within the normal ranges. Fine needle aspiration on the tumor revealed spindle shaped cells and a few gangliocytes without any evidence of malignancy, indicating that the tumor may be derived from retroperitoneal neural crest cells. 


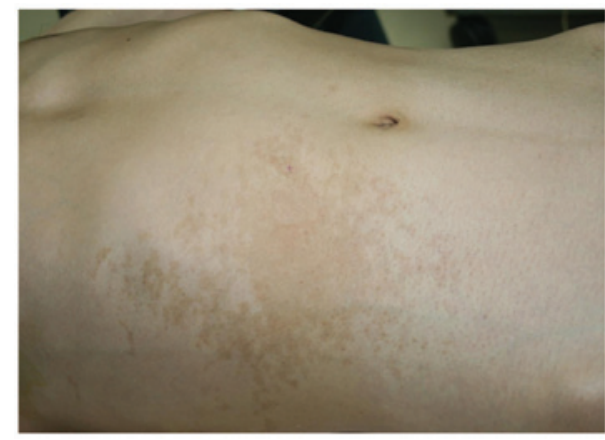

Figure 1. Café au lait macules (CALMs) in right upper quadrant and right hypochondriac region.

\section{A}
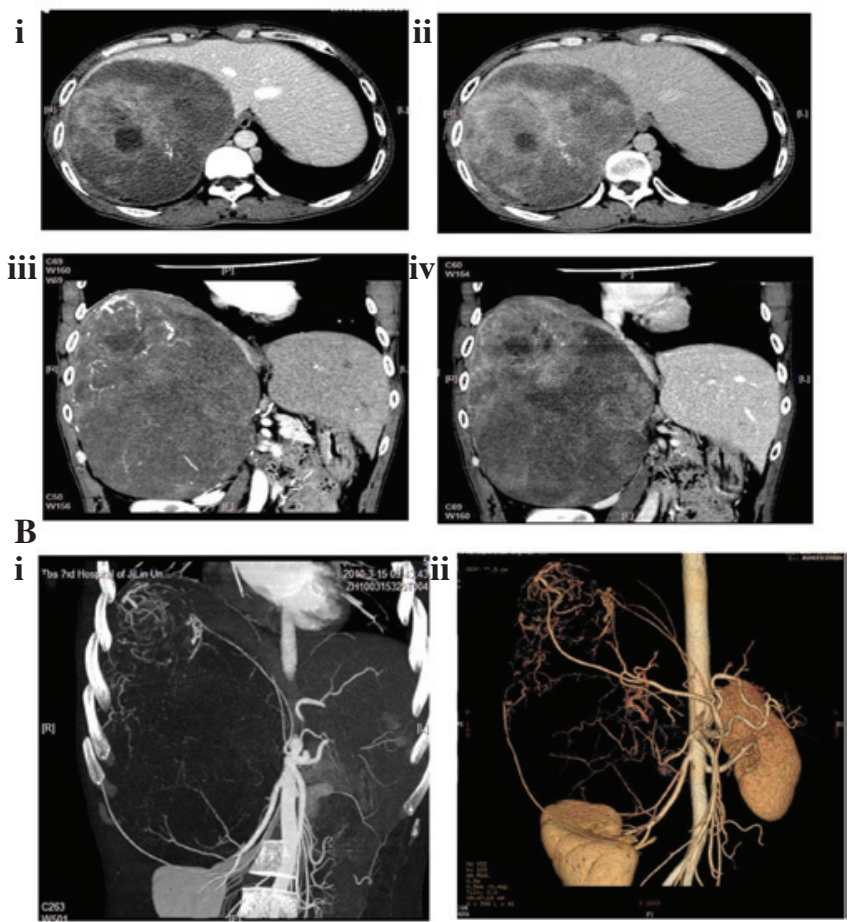

Figure 2. (Ai) CT scans showing that the tumor was well-defined, it occupied the right lobe of the liver and it was contrast-enhanced in the arterial phase. Necrosis and calcification were detected in the center of the enhanced regions. (ii) The tumor remained enhanced in the venous phase. (iii) Coronal scan in arterial phase. (iv) Coronal plane in venous phase illustrated that the hypervascular areas were located in the upper part of the tumor and had a close correlation with the right hepatic lobe. (Bi and ii) 3D CT vascular reconstruction revealed that the tumor was enriched through multiple blood supplies from the abdominal aorta, renal artery and hepatic artery. The middle to bottom part of the neoplasm was fed by the right renal and right lumber artery and the top by the right hepatic artery. There was a significantly high vascular density observed in the upper part of the neoplasm. CT, computed tomography.

An exploratory laparotomy was conducted, which demonstrated that the tumor originated from the retroperitoneal area of the abdominal cavity. It squeezed and compressed the right liver lobe (Fig. 3A) and a 1-cm metastatic nodule was observed in the compressed right liver lobe close to the tumor, which was separated with MPNST. The MPNST had a loose adhesion to adjacent retroperitoneal tissues and
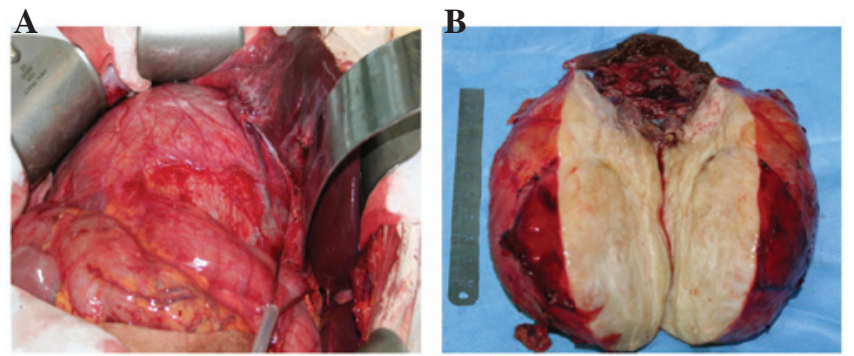

Figure 3. (A) Intraoperative findings of a giant, oblong retroperitoneal tumor with a tight connection to the right hepatic lobe. The right lobe of the liver was compressed and a metastatic lesion was detected in the right lobe of the liver close to the tumor. It was surrounded by a tenuous pseudocapsule. (B) Gross appearance of the tumor. Middle to bottom part appeared homogeneous and rich in connective tissue. The heterogeneous top part with necrosis and bleeding tightly adhered to the liver. A solid nodule of $1 \mathrm{~cm}$ diameter was observed in the liver with clear boundary.
A

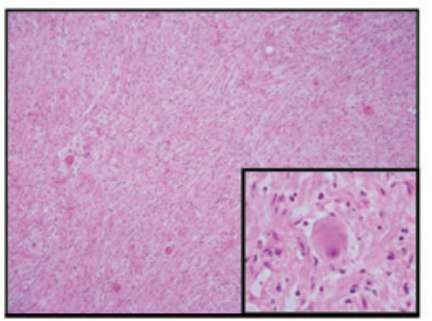

C

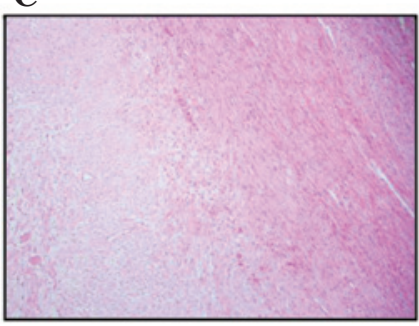

B

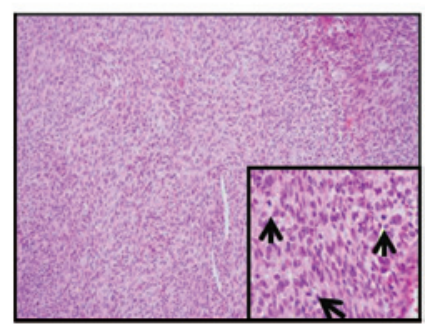

D

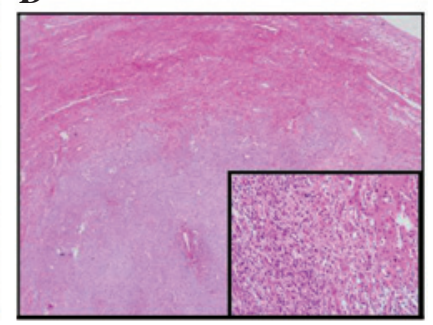

Figure 4. (A) The GN region consisted of sparse spindle cells scattered with mature gangliocytes (magnification, x200); insert showing an enlarged morphology of gangliocytes (magnification, x400). (B) Intensively arranged spindle cells with mitotic figures (magnification, x200); insert showing 2-4 mitotic cells per hpf, as indicated by arrows (magnification, $\mathrm{x} 400$ ). (C) There was a distinct boundary between benign and malignant regions. (magnification, $\mathrm{x} 200$ ) (D) Liver membrane nodule of the right lobe showing the liver metastasis (magnification, $\mathrm{x} 40$ ); insert showing malignant cells in the hepatic parenchyma (magnification, x400). GN, ganglioneuroma; hpf, high power field.

was resected completely with the partial right hepatic lobe (Fig. 3B).

Histological examination revealed a GN malignant transformation in which the tumor was composed of GN and MPNST. There was a line of demarcation between the two components. The GN region consisted of sparse spindle cells with scattered mature ganglioneurocytes (Fig. 4A). In addition, hyperchromatic spindle or short spindle cells intensively ranged in the malignancy demonstrating a fasciculated growth pattern in which 2-4 mitotic figures per high power field (hpf) were observed. Hemorrhage and necrosis in the malignant area were compatible with the hypodensity of the image (Fig. 4B). There was a distinct boundary between the benign 
and malignant regions (Fig. 4C) and malignant tumor cells were detected in the metastasized liver tissue (Fig. 4D).

Immunohistochemical staining revealed that the cells of the GN stained positive for S100 protein and syn, and the MIB-1 proliferative index (labeled with immunohistochemical stain for the Ki67 antigen) was $<1 \%$. In malignant tissue, CD56, GFAP, CD117, CD34, BCL-2 and P53 were stained positive, while S100, SMA and actin were stained negative. The MIB-1 proliferative index was $>40 \%$.

The postoperative course was uneventful and no additional therapy was administered. Since the procedure, the patient has been followed up closely and has been recurrence free for 4 months.

\section{Discussion}

GN is a rare, benign tumor arising from the neural crest cells. It is composited with ganglion cells, Schwann cells and a few neuroblasts. GN is most commonly located in the posterior mediastinum followed by the retroperitoneum, and is most frequently diagnosed between the ages of 10 and 40 years (4). Despite its extremely rare incidence, malignant transformations may occur and the neoplasm may exist in the form of an MPNST (5). The majority of GNs manifest as an asymptomatic mass, which are discovered on routine radiographic studies for other lesions. The present case was symptomatically associated with pain of the abdomen and metastasis to the liver. Using contrast-enhanced CT, we have demonstrated that multiple blood supplies from the abdominal aorta, renal artery and hepatic artery may be the cause of quick tumor progression, malignant transformation and abdominal symptoms of the tumor.

MPNST is rare, with an expected incidence of $0.001 \%$ (6). It has been suggested that the disease occurrence is usually associated with radiation or neurofibromatosis type 1 (NF-1). However, how much of those factors contribute to the occurrence of MPNST and whether the ethnicity and region are involved are unknown. Li et al and Ye et al reported that a few patients had a history of radiotherapy or neurofibromatosis $(7,8)$. Coffin and Dehner (9) have reported that retroperitoneal malignant schwannoma in the absence of NF-1 is extremely rare. The definite correlation of the regional diversity in the incidence of MPNST remains uncertain. The present case has no evidence of NF-1 or radiation history, but regional skin brown pigmentation was identified on the right hypochondric region of the abdomen. Over the years, a universal notion has developed which suggests that GN patients with NF-1 have a greater lifetime risk of developing MPNST and tend to have a worse prognosis. de Chadarevian et al (2) reviewed 12 fully documented MPNST patients arising from GN of 139 MPNST cases. Similar to the presentation of our patient, none of the cases had NF, indicating that other factors may involved.

Our histological examination revealed that the tumor included two distinct components: benign loose texture areas and malignant compact texture areas with necrosis and hemorrhage. The majority of the middle to bottom area of the tumor consisted of benign, well-differentiated spindle cells with scattered mature ganglion cells, while the malignant region located at the top part of the tumor extruded into the hepatic parenchyma. The lesion detected in the liver had its own boundary and ganglion cells were revealed inside. Based on these histological results, the tumor was composed of approximately $75 \%$ GN and 25\% MPNST. Abrupt transition of the two different components was observed. We concluded that MPNST arose from the transformation of GN since ganglion cells were identified in the malignant area. However, this does not preclude the conceptual possibility that any type of nerve sheath cells are able to turn into malignant neoplasms. Therefore, possible initiators are either Schwann cells recruited by the GN or one of the integral neoplastic constituents of the benign GN. Studies on how MPNST may be derived from GN are required (10) as only limited data are available regarding the research of this transformation.

MPNST is usually asymptomatic, however, symptoms may occur in certain cases depending on the location of the tumor and whether the hormones are being secreted or not. MPNSTs have few laboratory characteristics and morphological features, making the preoperative diagnosis difficult. In almost all cases, the diagnosis depends on the pathology and immunohistochemistry examinations. In our patient, there was an important physical sign of abdominal café au lait macules (CALMs), which was nearly overlooked. CALM belongs to the definition of neurocutaneous syndrome. Skin lesions may occur sporadically in the general population and are nearly universal findings in patients with NF-1. However, there have been no publications reporting objective quantification of CALM pigmentation (11).

Arteriography and 3D CT vascular reconstruction may be useful to visualize the vascularization. Manifesting the artery blood supply is generally the most reliable method for ruling out the origin of the tumors. In our patient, the nutritious blood originated from multiple arteries involving the branches of the renal artery, right hepatic artery and abdominal aorta. The fact that the blood supply by hepatic vessels to the top part of the tumor may be a result from hepatic invasion, confirms the vascular connection between the liver and the tumor. Rapid proliferation of malignant cells usually occurs in a relatively ischemic situation, which leads to the compensation of blood supply from the liver. This phenomenon is pathophysiologically reasonable; however, there are no previous studies on uncommon blood supplies in MPNST cases. This has made it difficult to differentiate the tumor from HCC until surgery.

The prognosis of MPNST has been poorly reported. Our patient received a complete surgical excision and was recurrence free for only 4 months. MPNST is not sensitive to radiation or chemotherapy, so there are no effective therapeutic methods to be used postoperatively (12). In the perspective of our case and accumulated reports of GN malignant transformations to MPNST, although extremely rare, further systematic investigations are feasible and important to elucidate the mechanisms of GN and MPNST.

\section{References}

1. Jung HR, Kang KJ, Kwon JH, and Kang YN: Adrenal ganglioneuroma with hepatic metastasis. J Korean Surg Soc 80: 297-300, 2011.

2. de Chadarevian JP, Maepascasio J, Halligan GE, Katz DA, Locono JA, Kimmel S and Katsetos CD: Malignant peripheral nerve sheath tumor arising from an adrenal ganglioneuroma in a 6-year-old boy. Pediatr Dev Pathol 7: 277-284, 2004. 
3. Okada K, Hasegawa T, Tajino T, et al: Clinical relevance of pathological grades of malignant peripheral nerve sheath tumor: a multi-institution TMTS study of 56 cases in Northern Japan. Ann Surg Oncol 14: 597-604, 2007.

4. Gary C, Robertson H, Ruiz B, Zuzukin V and Walvekar RR: Retropharyngeal ganglioneuroma presenting with neck stiffness: report of a case and review of literature. Skull Base 20: 371-374, 2010.

5. Acin-Gandara D, Carabias A, Bertomeu A, Gimenez-Alvira L, Colao L and Limones M: Giant retroperitoneal ganglioneuroma. Rev Esp Enferm Dig 102: 205-207, 2010 (In Spanish).

6. Chhabra A, Soldatos T, Durand DJ, Carrino JA, McCarthy EF and Belzberg AJ: The role of magnetic resonance imaging in the diagnostic evaluation of malignant peripheral nerve sheath tumors. Indian J Cancer 48: 328-334, 2011.

7. Li C, Shi Y, Luo H and Wang J: Giant retroperitoneal malignant schwannoma: a case report and review of literature. Chinese-German J Clinical Oncol 9: 180-182, 2010.
8. Ye TS, Zhang XF, Wang Y, et al: Clinical research of malignant peripheral nerve sheath tumor (36 cases report). Med J Chin PLA 8: 717-718, 2003 (In Chinese).

9. Coffin CM and Dehner LP: Peripheral neurogenic tumors of the soft tissues in children and adolescents: a clinicopathologic study of 139 cases. Pediatr Pathol 9: 387-407, 1989.

10. Mora J, Cheung NK, Juan G, et al: Neuroblastic and Schwannian stromal cells of neuroblastoma are derived from a tumoral progenitor cell. Cancer Res 61: 6892-6898, 2001.

11. Boyd KP, Gao L, Feng R, Beasley M, Messiaen L, Korf BR and Theos A: Phenotypic variability among café-au-lait macules in NF-1. J American Acad Dermatol 63: 440-447, 2010.

12. Anghileri M, Miceli R, Fiore M, Mariani L, Ferrari A, Mussi C, Lozza L, Collini P, Olmi P, Casali PG, Pilotti S and Gronchi A: Malignant peripheral nerve sheath tumors: prognostic factors and survival in a series of patients treated at a single institution. Cancer 107: 1065-1074, 2006. 